\title{
NEAR-IR POLARIMETRY AROUND 30 DORADUS: I. SEPARATION OF THE GALACTIC SOURCES
}

\author{
Jaeyeong Kim ${ }^{1}$, Soojong Pak ${ }^{1}$, Minho $\mathrm{Chol}^{2}$, Wonseok $\mathrm{Kang}^{1}{ }^{3}$ Ryo Kandori ${ }^{3}$, Motohide Tamura ${ }^{3}$, Tetsuya \\ NAGATA $^{4}$, Jungmi KWON ${ }^{3}$, DAisuke KATO ${ }^{5}$, AND DANiEl T. JAFFe ${ }^{1,6}$ \\ 1 School of Space Research, Kyung Hee University, 1 Seocheon-dong, Giheung-gu, Yongin, Gyeonggi-do 446-701, \\ Korea \\ E-mail : jaeyeong@khu.ac.kr,soojong@khu.ac.krandwskang@khu.ac.kr \\ 2 Korea Astronomy and Space Science Institute, 776 Daedeokdaero, Yuseong, Daejeon 305-348, Korea \\ E-mail : minho@kasi.re.kr \\ ${ }^{3}$ National Astronomical Observatory of Japan, Mitaka, Tokyo 181-8588, Japan \\ E-mail : r.kandori@nao.ac.jp, motohide.tamura@nao.ac.jpand jmkwon@khu.ac.kr \\ ${ }^{4}$ Department of Astronomy, Kyoto University, Kyoto 606-8502, Japan \\ E-mail : nagata@kusastro.kyoto-u.ac.jp \\ ${ }^{5}$ Institute of Space and Astronomical Science, Japan Aerospace Exploration Agency, 3-1-1 Yoshinodai, Chuo-ku, \\ Sagamihara, Kanagawa, 252-5210, Japan \\ E-mail : kato@ir.isas.jaxa.jp \\ ${ }^{6}$ Department of Astronomy, University of Texas at Austin, 1 University Station, Austin, TX 78712-0259, USA \\ E-mail : dtj@astro.as.utexas.edu
}

(Received January 7, 2011; Revised August 1, 2011; Accepted August 2, 2011)

\begin{abstract}
A $20^{\prime} \times 20^{\prime}$ region around 30 Doradus in the Large Magellanic Cloud (LMC) is observed and analyzed in the near-infrared. We obtain polarimetry data in the $J, H$, and $K_{s}$ bands using the SIRIUS polarimeter SIRPOL at the Infrared Survey Facility $1.4 \mathrm{~m}$ telescope. We measure the Stokes parameters of 2562 point-like sources to derive the degree of polarization and the polarization position angles. We discuss the statistics of the groups classified by color-magnitude diagram and proper motions of the sources, in order to separate the Galactic foreground sources from those present in the LMC. We notice that groups classified by the proper motion data show a tendency towards different polarimetric properties.
\end{abstract}

Key words : techniques: polarimetric — infrared: ISM — galaxies: star clusters: individual (30 Doradus) — proper motions

\section{INTRODUCTION}

The role of magnetic fields is relevant in star formation processes and in galaxy evolution science (Kepley 2009; Schmidt 1970, 1976; Mathewson \& Ford 1970; Wielebinski 1995). Measuring the orientation of dust grains is a way to investigate the physical properties of the magnetic field, since the magnetic field makes dust grains to align, due to rapid precession enforced from the substantial magnetic moment (Davis \& Greenstein 1951; Dolginov \& Mytrophanov 1976). In star-forming molecular clouds, dust grains tend to be aligned with their long axes perpendicular to the magnetic field (Lazarian 2006); dichroic polarization in the optical and near-IR wavelengths can measure the direction of those fields (Davis \& Greenstein 1951). In addition, the statistical pattern of the polarization position angles allows one to quantify the magnetic field strength (Ostriker et al. 2001; Kwon et al. 2010).
The Large Magellanic Cloud (LMC) is one of the nearest external galaxies. The interstellar medium of the LMC shows quite different physical and chemical properties with respect to that of our Galaxy, e.g., lower metallicity and different dust properties (Pak et al. 1998; Nakajima et al. 2007; Kim et al. 2010). The LMC has revealed large-scale magnetic field structures (Gaensler et al. 2005) from optical polarization observations (Schmidt 1970, 1976; Mathewson and Ford 1970; Wayte 1990). These features indicate that, in the west side of the LMC, the magnetic field is pointing towards the Small Magellanic Cloud. On the other hand, the magnetic fields around 30 Doradus appear to be complex structures. In fact, 30 Doradus is a unique, giant star formation complex in the LMC and contains massive clusters (Walborn \& Blades 1997; Brandner et al. 2001; Maercker \& Burton 2005; Scowen et al. 2009). Multi-frequency radio continuum surveys (Haynes et al. 1991) show the presence of filamentary magnetic fields in the LMC.

For this study, we observed near-infrared (NIR) 


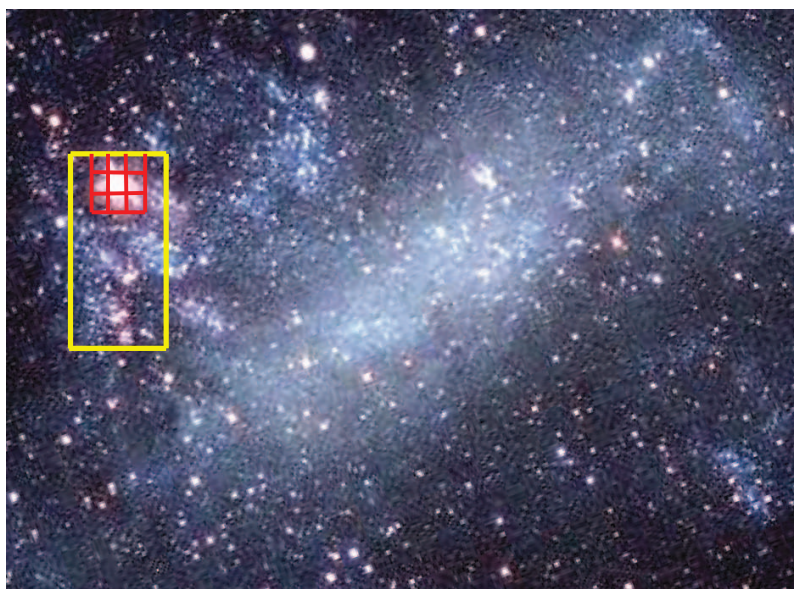

Fig. 1.- Observed area of the LMC on an optical image offered by Mr. Kamiya. The yellow box shows the total $5 \times 9$ survey field by SIRPOL on 2008 December 25 and 30 and the red box is the $3 \times 3$ fields around 30 Doradus.

sources in star forming regions $\left(\sim 39^{\prime} \times 69^{\prime}\right)$ in the LMC. The number of detected sources, however, is quite large. In this paper, we only present preliminary results of selected regions around 30 Doradus $\left(\sim 20^{\prime} \times 20^{\prime}\right)$. Section 2 describes the observations and the data reduction processes, including photometry and the derivation of the Stokes parameters. Section 3 includes the comparison of our data with previous observations by Nakajima et al. (2007). In Section 4, we discuss the methods to extract Galactic foreground sources using the color-magnitude diagram and proper motions.

\section{OBSERVATIONS AND DATA REDUC- TION}

The observations were carried out at the Infrared Survey Facility (IRSF) $1.4 \mathrm{~m}$ telescope in the South African Astronomical Obsevatory on December 25 and 30, 2008. Using the infrared camera SIRIUS (Nagayama et al. 2003) and the polarimeter SIRPOL (Kandori et al. 2006), we observed regions around 30 Doradus in $J(1.25 \mu \mathrm{m}), H(1.63 \mu \mathrm{m})$, and $K_{s}(2.14$ $\mu \mathrm{m})$ bands. This system enables us to obtain a widefield $(7 ! 7 \times 7 ! 7)$ polarimetric image with a scale of $0 . ! 45$ pixel $^{-1}$.

We performed four 20-second exposures at waveplate angles of $0^{\circ}, 45^{\circ}, 22^{\circ} .5,67^{\circ} .5$, and at 10 dithered positions for each field position. The total integration time was 200 seconds per wave-plate angle. The typical seeing size was $\sim 1^{\prime \prime} 3$ in the $J$ band, but we experienced unstable seeing conditions during a night. Among the observed $5 \times 9$ fields $\left(\sim 39^{\prime} \times 69^{\prime}\right)$ we analyzed the central $3 \times 3$ fields $\left(\sim 20^{\prime} \times 20^{\prime}\right)$ and illustrate here our initial investigations (see Fig. 1). Table 1 lists the observation $\log$ of the data presented in this paper.

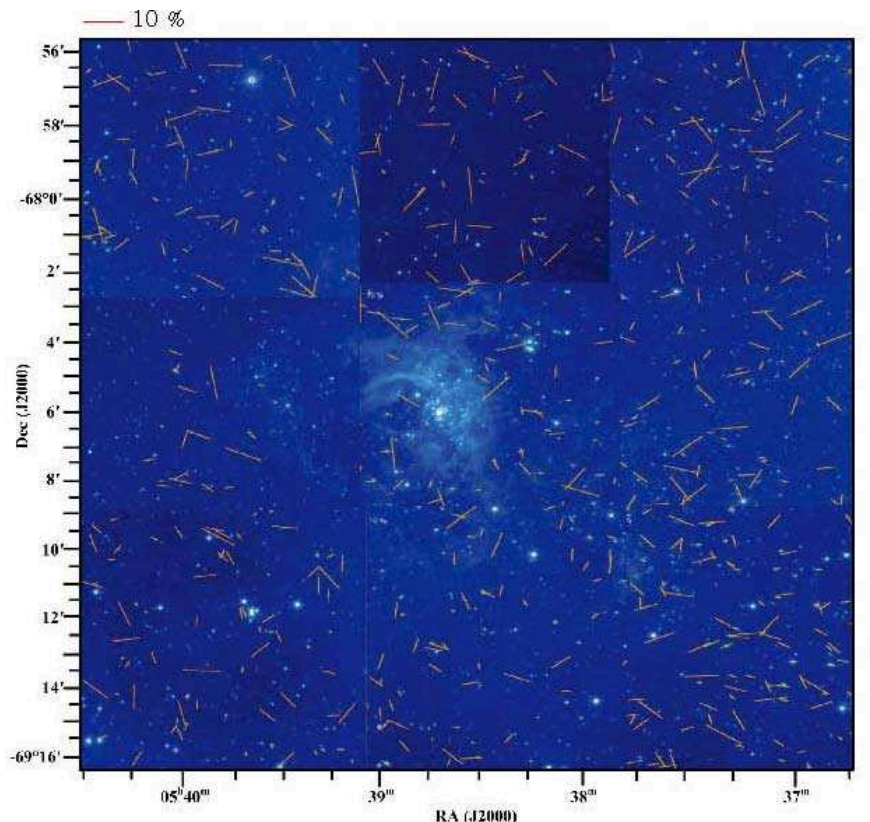

Fig. 2. $-H$ band intensity image of the $3 \times 3$ fields and polarization vectors (orange lines) of the polarized sources in the $H$ band. The length of the vectors indicates the polarization degree (\%).

We used the IRAF* (Image Reduction \& Analysis Facility) software package to reduce the data, e.g., dark-field subtraction, flat-field correction and median sky subtraction. In addition, the data for each waveplate position $\left(I_{0}, I_{45}, I_{22.5}\right.$ and $\left.I_{67.5}\right)$ were processed as described in Kandori et al. (2006). For source detection and photometry on the Stokes $I$ images, we used the IRAF daophot package (Stetson 1987). We set the aperture radius to 8 pixels and the sky annulus to 13 pixels, and detected point sources having peak intensity greater than $10 \sigma$ above the local sky background. The pixel coordinates of the detected sources were matched to the celestial coordinates of their counterparts in the $2 \mathrm{MASS}^{\dagger}$ Point Source Catalogue. The magnitude and color of our photometry were transformed into the 2MASS system using:

$$
\begin{gathered}
m_{2 M A S S}=m_{I R S F}+\alpha_{1} \times C_{I R S F}+\beta_{1}, \\
C_{2 M A S S}=\alpha_{2} \times C_{I R S F}+\beta_{2},
\end{gathered}
$$

where $m_{I R S F}$ is the instrumental magnitude from the

\footnotetext{
* IRAF is distributed by the US National Optical Astronomy Observatories, which are operated by the Association of Universities for Research in Astronomy, Inc., under cooperative agreement with the National Science Foundation.

${ }^{\dagger}$ The Two Micron All Sky Survey(2MASS) is a joint project of the University of Massachusetts and the Infrared Processing and
} Analysis Center/California Institute of Technology. 
Table 1.

Observation Log

\begin{tabular}{lcccc}
\hline \hline Date(LT) & Field name & $\alpha_{\text {○J2000.0 }}$ & $\delta_{\text {○J2000.0 }}$ & Seeing in J band $\left[^{\prime \prime}\right]$ \\
\hline \multirow{2}{2}{ 2008 Dec 25 } & $\mathrm{n} 4$ & 053719.86 & -690603.3 & 1.4 \\
& $\mathrm{n} 5$ & 053834.48 & -690602.2 & 1.4 \\
& $\mathrm{n} 6$ & 053948.78 & -690602.6 & 1.3 \\
& $\mathrm{n} 7$ & 053948.17 & -691238.0 & 1.3 \\
& $\mathrm{n} 8$ & 053832.91 & -691234.0 & 1.2 \\
& $\mathrm{n} 9$ & 053717.79 & -691232.4 & 1.3 \\
\hline \multirow{2}{*}{2008 Dec 30 } & $\mathrm{n} 1$ & 053947.92 & -685909.9 & 1.2 \\
& $\mathrm{n} 2$ & 053833.53 & -685907.7 & 1.3 \\
& $\mathrm{n} 3$ & 053719.27 & -685903.9 & 1.3 \\
\hline
\end{tabular}
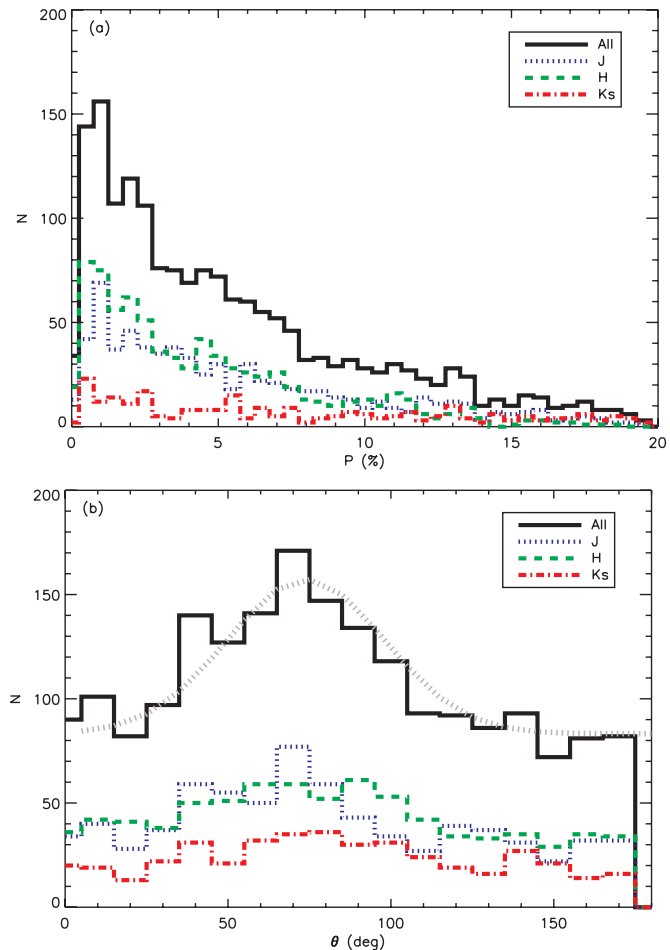

Fig. 3.- (a) Histograms of polarization degree of the sources in nine fields with $P \geq 3 \sigma_{P}$ for the $J, H$, and $K_{s}$ bands. (b) Histograms of polarization angle of the sources in nine fields with $P \geq 3 \sigma_{P}$ for the $J, H$, and $K_{s}$ bands. The grey dotted line on the histogram of the all sources show the results of Gaussian fits. The central angle and the sigma of the Gaussian function are listed in Table 2.

IRSF images and $m_{2 M A S S}$ is the magnitude from the 2MASS All Sky Point Source Catalogue.

We calculated the Stokes parameters $I, Q$, and $U$, the polarization degree $P$, and the polarization position angle $\theta$, as follows:

$$
\begin{gathered}
I=\left(I_{0}+I_{22.5}+I_{45}+I_{67.5}\right) / 2, \\
Q=I_{0}-I_{45}, \\
U=I_{22.5}-I_{67.5}, \\
P_{\circ}=\frac{\sqrt{Q^{2}+U^{2}}}{I}, \\
\theta=\frac{1}{2} \arctan \frac{U}{Q} .
\end{gathered}
$$

Since the initial polarization degree $P_{\circ}$ is a positive quantity, the derived $P_{\circ}$ values tend to be overestimated, especially for low signal-to-noise ratio $(\mathrm{S} / \mathrm{N})$ sources. To correct for the bias, we use the following equation:

$$
P=\sqrt{P_{\circ}^{2}-\delta P_{\circ}^{2}},
$$

where $\delta P_{\circ}$ is the error in $P_{\circ}$ (Wardle \& Kronberg 1974). The polarization efficiencies of SIRPOL (95.5, 96.3, and $98.5 \%$ at $J, H$, and $K_{s}$ bands, respectively) were also used for correcting the polarization degree (Kandori et al. 2006).

\section{RESULTS}

From the data reduction process, we first derive polarization degrees and angles of 2562 sources, and extract only those with $P \geq 3 \sigma_{P}$. ( $\sigma_{P}$ indicates the error of a polarization value.) The numbers of the selected stars are 736,784 , and 427 in $J, H$, and $K_{s}$ bands, respectively. Fig. 2 shows a polarization vector map in the reduced 9 fields. The statistical distribution of the polarization degrees and angles is shown in Fig. 3 .

To confirm our results, we compare our data with previous observations. Nakajima et al. (2007), hereafter N07, had observed the central $7 ! 7 \times 7 ! 7$ (one field) regions of 30 Doradus using the same SIRIUS/SIRPOL system. Our photometry in the 30 Doradus is limited 

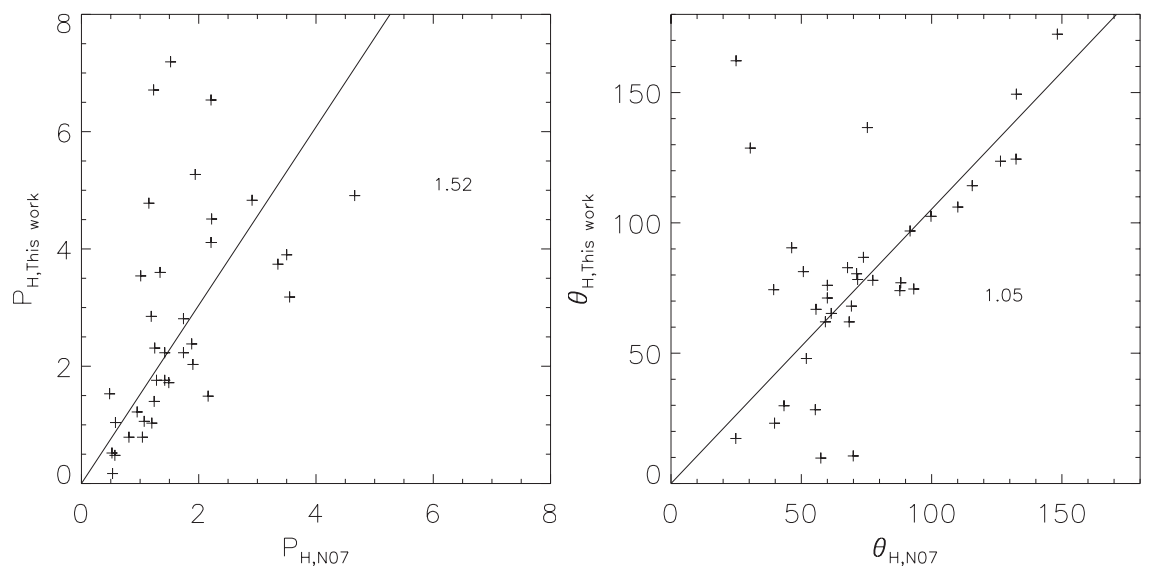

Fig. 4. - Correlation graph of matched data in the $H$ band. Polarization degree (left) and angle (right) are shown. The solid lines are linear fits with slopes of 1.52 (left) and 1.05 (right).

Table 2.

Statistical Properties of the Polarization angle

\begin{tabular}{ccccc}
\hline \hline & $J^{a}$ & $H^{a}$ & $K_{s}{ }^{a}$ & All bands $^{a}$ \\
\hline All sources & $79 \pm 47$ & $82 \pm 48$ & $85 \pm 46$ & $74 \pm 24$ \\
& & & & \\
\hline GroupB & $75 \pm 49$ & $83 \pm 53$ & $75 \pm 43$ & $76 \pm 11$ \\
GroupEFG & $81 \pm 47$ & $85 \pm 47$ & $87 \pm 49$ & $73 \pm 21$ \\
\hline$\mu>2 \sigma_{\mu}$ & $90 \pm 45$ & $102 \pm 51$ & $97 \pm 44$ & $96 \pm 19$ \\
$\mu<2 \sigma_{\mu}$ & $69 \pm 46$ & $82 \pm 48$ & $83 \pm 46$ & $65 \pm 24$ \\
\hline
\end{tabular}

Note. — ${ }^{a}$ Polarization angle in degree units. The central angle and the sigma values are from obtained Gaussian fits to the distribution functions.

by the nebula features, while N07 detected many point sources with long exposures, i.e., a total of 1480 seconds per wave-plate angle.

The numbers of the matched sources in both data are 33,36 , and 7 , at $J, H$, and $K_{s}$ bands, respectively. With these sources, we compared the polarization degree and angle. Figure 4 shows that our values of the polarization angles and those from N07 are somehow consistent. However, the polarization degree values in our works are $50 \%$ larger than those in N07. We suspect that this discrepancy is caused by the underestimated $\delta P_{\circ}$ values in our low $\mathrm{S} / \mathrm{N}$ data. More observations and analysis is required to confirm the calibration of the polarization degree values.

\section{DISCUSSION}

Source classification is important to separate Galactic foreground sources from those in the LMC, because they can contaminate polarimetry by their intrinsic polarization. In this section, we applied two methods to test the separation reliabilities.

\subsection{Color-Magnitude Diagram}

The typical classification method is to use colormagnitude diagrams (hereafter $C M$ diagrams). Nikolaev \& Weinberg (2000), hereafter NW00, classified the LMC stars using 2MASS data as follows:

- Group A: blue supergiants and O dwarfs in the LMC; - Group B: Galactic disk dwarfs with spectral classes of $\mathrm{F}-\mathrm{K}$ and Galactic giants with spectral classes of $\mathrm{F}-\mathrm{G}$;

- Group D: Galactic disk dwarfs with spectral classes 


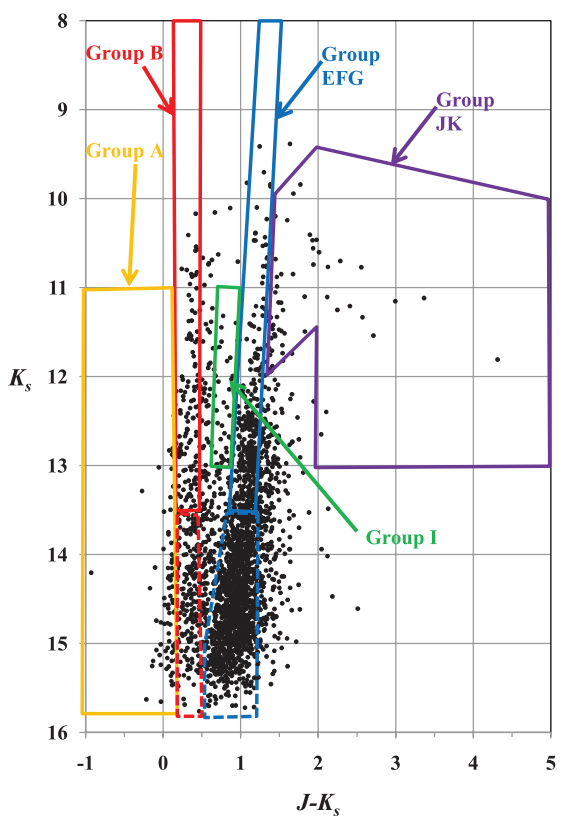

Fig. 5. - Color-magnitude $\left(J-K_{s}\right.$ vs. $\left.K_{s}\right)$ diagram of our sources. Each group is indicated with a different color box: Group A (orange box), Group EFG (blue box), Group B (red box), Group I (green box), and Group JK (violet box). Our photometry data can detect more faint sources $\left(m_{K_{s}}\right.$ $<16 \mathrm{mag}$ ) than the previous data of Nikolaev \& Weinberg (2000) and most of the sources are distributed in the range of $14<m_{K_{s}}<16 \mathrm{mag}$. The red and blue dashed boxes are extrapolated regions from Group B and Group EFG.

of $\mathrm{G}-\mathrm{M}$, red-giant branch (RGB), and early asymptotic giant branch (E-AGB) stars in the LMC;

- Group EFG: tip of the RGB, E-AGB, and O-rich AGB stars in the LMC ;

- Group I: super giants and the red clump in the LMC;

- Group JK: C-rich AGB and dusty AGB stars in the LMC.

The 2MASS sources, however, are limited in $m_{K_{s}}$ $<14$ mag. On the other hand, our photometry data cover up to $m_{K_{s}}<16 \mathrm{mag}$ and most detected sources are populated under Group D in NW00. Kato et al. (2007) used the criteria of NW00, but did not classify the sources which distributed under Group D. We followed the same criteria as Kato et al. (2007) to separate sources. Fig. 5 shows our five groups.

Galactic foreground stars are mostly distributed above the brighter region $\left(m_{K_{s}}<11\right)$ of Group $A$ according to NW00. However, our data do not contain the detected sources in that region. The detected foreground sources are distributed in Group B; the Galactic giants with spectral classes of $\mathrm{F}-\mathrm{G}$ have a small population $(\leq 5 \%)$ within this group. Most RGB and AGB stars in the LMC are in Group EFG. Note that Group $A$ and Group I can be confused with the Galactic foreground sources (Group B) be-
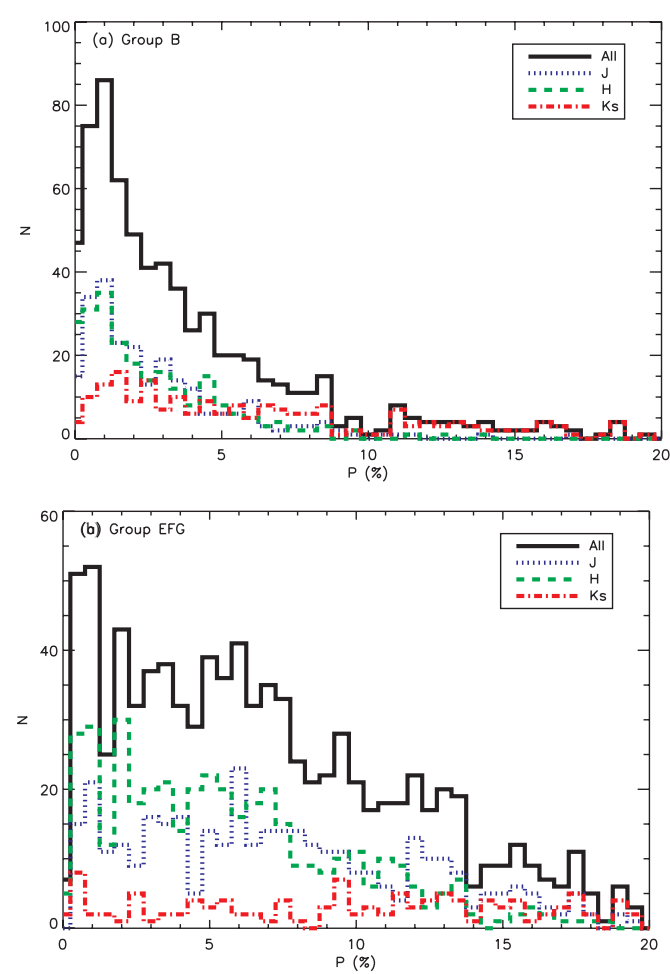

Fig. 6. - (a) Histograms of the polarization degree of the sources which are classified to the Group $B$ for the $J, H$, and $K_{s}$ bands. (b) Histograms of the polarization degree of the sources which are classified to the Group EFG and $P$ $\geq 3 \sigma_{P}$ for the $J, H$, and $K_{s}$ bands.

cause of their uncertain boundary lines. Group JK have dusty AGB stars which may have their own polarization by the shrouded dust shells (Parthasarathy \& Jain 1993). Therefore, Group A, Group I and Group $J K$ are excluded from the samples when comparing the foreground and the main LMC sources.

We classify Group $B$ as foreground sources and Group EFG as the main LMC stars. We extrapolate the boundaries of the criteria regions up to $m_{K_{s}}<16$ mag and plot histograms for the sources in Group $B$ and Group EFG. Fig. 6 and 7 show the distributions of polarization degrees and angles for both Group $B$ and Group EFG. In Fig. 6, the polarization degree distribution of Group EFG shows a large population of high polarization values $(P>10 \%)$ which comes from the intrinsic polarization of the RGB and AGB stars. On the other hand, the polarization angle distributions show similar tendency. See the statistical distributions in Fig. 7 and Table 2. Because the criteria of Group $B$ and Group EFG are overlapped in $14<m_{K_{s}}<$ 16 , we expect that many sources may not be classified properly. 

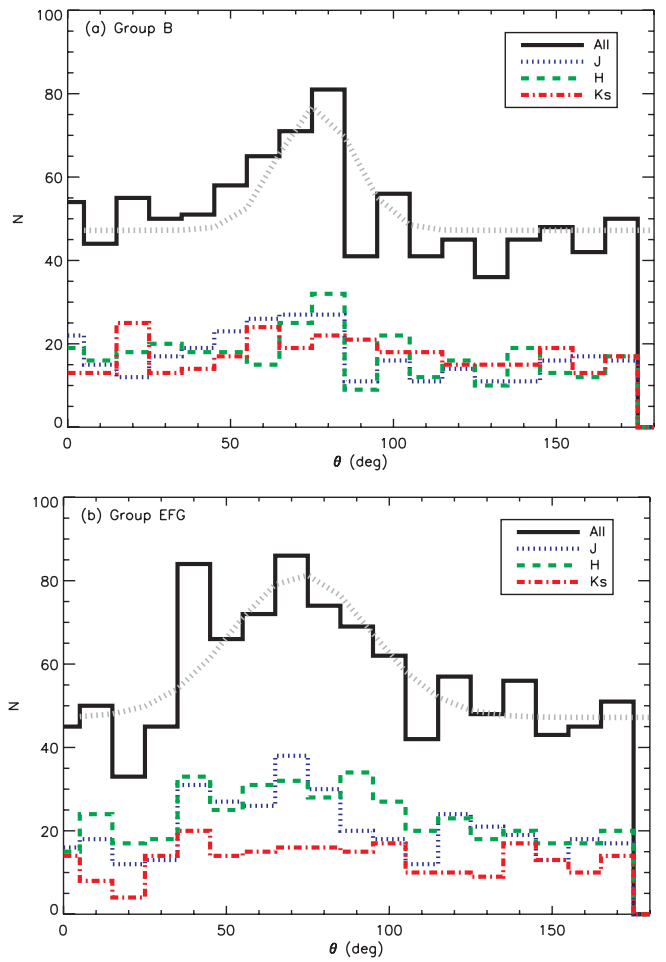

Fig. 7.- (a) Histograms of the polarization angle of the sources which belong to the Group $B$ for the $J, H$, and $K_{s}$ bands. (b) Histograms of the polarization angle of the sources which belong to the Group EFG and $P \geq 3 \sigma_{P}$ for the $J, H$, and $K_{s}$ bands.

\subsection{Proper Motion}

Vieira et al. (2010) investigated the proper motion of the Magellanic Clouds from the resulting catalog of 1.4 million objects in the Southern Proper Motion (SPM) program. They used the proper motion of 3822 and 964 sources near the clouds to derive the mean absolute proper motions of the LMC and of the SMC. We used this catalog and examined proper motions to separate the foreground sources which would be contained in our observed regions in the LMC. A total of 805 sources were matched with SPM sources in the $J H K_{s}$ bands. Vieira et al. (2010) discarded areas of high stellar density, at $4^{h} 40^{m} 58.8^{s} \leq \alpha \leq 5^{h} 40^{m} 12^{s}$ and $-68^{\circ} \leq \delta \leq-71^{\circ}$, to avoid the misidentified measurement. Since our observed regions are located on the edge of the discarded area, some of our data may not be reliable.

In order to reduce the uncertainty in the data, we used data which had proper motions with $\mu>2 \sigma_{\mu}$ to select Galactic foreground stars. Fig. 8 shows the distribution of the sources according to the $\mathrm{S} / \mathrm{N}$ of the proper motion. Based on the existence of proper motion, we examined the difference between the LMC sources and the Galactic foreground ones.

Fig. 9 shows that the histograms of the polariza- tion degrees are similar between the two groups, while those of the $C M$ diagram classifications in Figure 6 are very different. We expect that the difference in Fig. 6 is partly from the intrinsic differences of the spectral types. As evident from Fig. 10, the polarization angle distributions are slightly different. The Galactic foreground group with proper motions shows almost equally distributed angles with a slight peak at $93^{\circ}$, while that of the LMC group shows a peak at $75^{\circ}$.

We also examined the possibility that the Galactic foreground stars may show some polarization properties different from the main LMC stars by their intrinsic polarization or the effect for the Galactic magnetic fields. Previous observations (Mathewson \& Ford 1970; Isserstedt \& Reinhardt 1976; Schmidt 1976) indicated that the Galactic magnetic fields at the line of sight of the LMC can influence the polarization properties of the foreground stars. According to those papers, the polarization properties of the Galactic sources in front of the LMC have a $0.3 \%$ polarization degree and $30^{\circ}$ of polarization angle. However, these results may not be applied to ours because our detection limits are too low to measure the dichroic polarization of the Galaxy.

\section{SUMMARY}

We conducted imaging polarimetry of the $3 \times 3$ fields $\left(\sim 20^{\prime} \times 20^{\prime}\right)$ around 30 Doradus. Because of unstable seeing condition, our aperture radius for photometry and polarimetry has been set $2-3$ times larger than the usual aperture radius. We used some previous data to confirm our results. Comparing with the sources of N07, we found no significant differences in our polarimetry data.

We applied several methods to remove the Galactic foreground sources which can contaminate the polarimetry result by their intrinsic polarization. Using the $C M$ diagram, we selected the group which contains the foreground sources. The main LMC sources show larger polarization degrees than the foreground sources.

We also selected the Galactic foreground sources using proper motion data. The foreground sources show high values of proper motion, contrary to the main LMC sources.

Comparing different proper motions, we can separate out the Galactic foreground sources from the main LMC sources. They showed different distributions for polarization degrees and angles with respect to the main LMC sources.

\section{ACKNOWLEDGMENTS}

J. Kim was supported by a WCU (World Class University) program through the National Research Foundation of Korea funded by the Ministry of Education, Science and Technology (R31-10016). Part of this work was supported by the National Research Foundation of Korea funded by the Ministry of Education, Science and Technology No. 2009-0063616. 


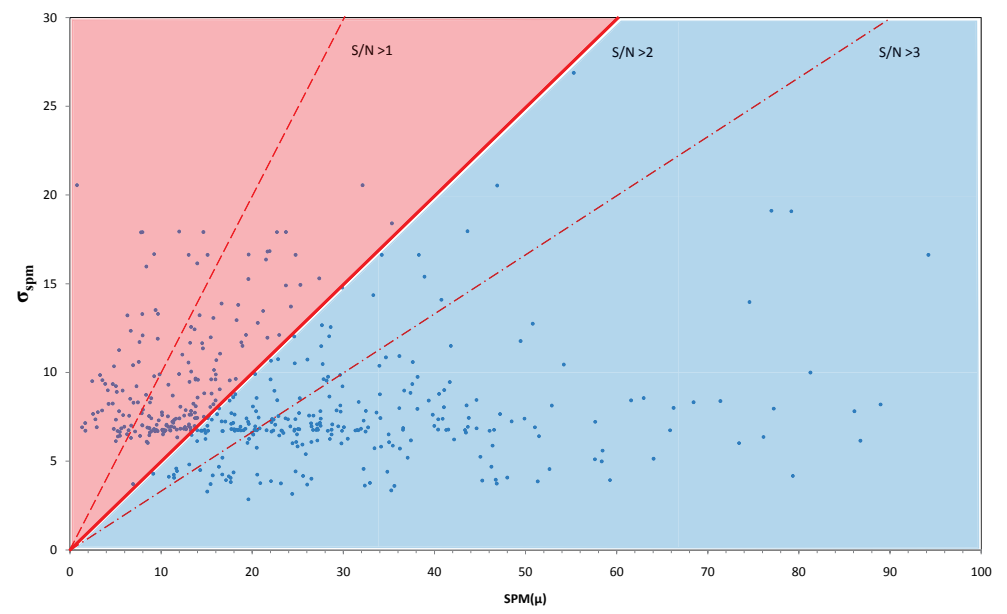

Fig. 8. - Plots of the proper motion and of its error. The blue region $(\mathrm{S} / \mathrm{N}>2)$ may contain most of the foreground sources, and the main LMC sources are mostly distributed in the red region $(\mathrm{S} / \mathrm{N}<2)$. From the $\mathrm{S} / \mathrm{N}$ ratio of the proper motion we try to separate sources.
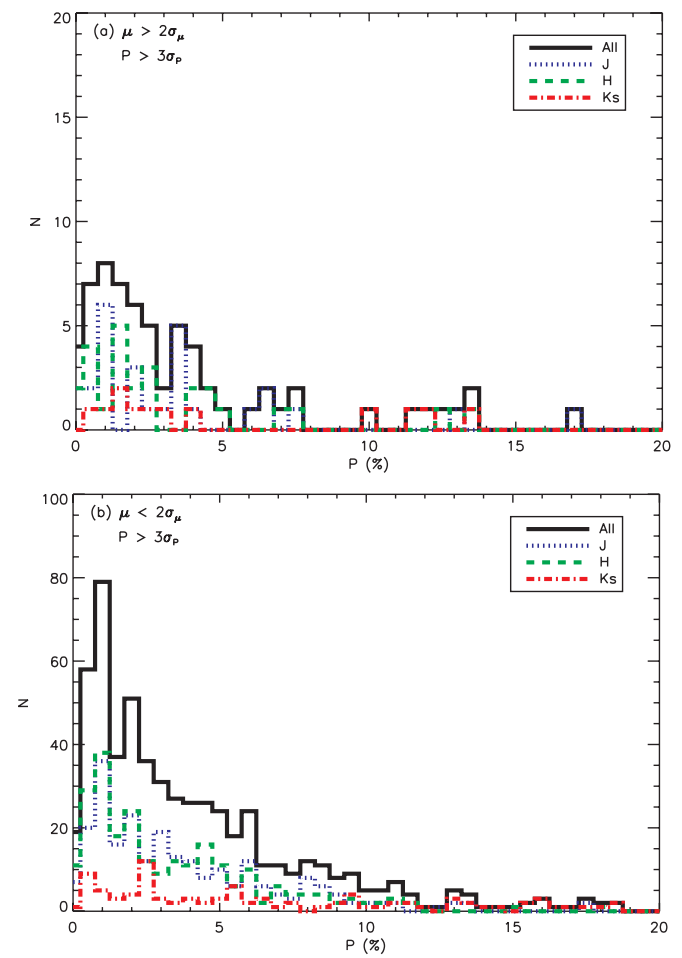

Fig. 9.- (a) Histograms of the polarization degree of the sources which have proper motion $\mu>2 \sigma_{\mu}$ and $P>3 \sigma_{P}$ for the $J, H$, and $K_{s}$ bands. (b) Histograms of the polarization degree of the sources which have proper motion $\mu<2 \sigma_{\mu}$ and $P>3 \sigma_{P}$ for the $J, H$, and $K_{s}$ bands.

\section{REFERENCES}

Brandner, W., Grebel, E. K., Barbá, R. H., Walborn, N. R., \& Moneti, A. 2001, Hubble Space Telescope NICMOS Detection of a Partially Embedded,
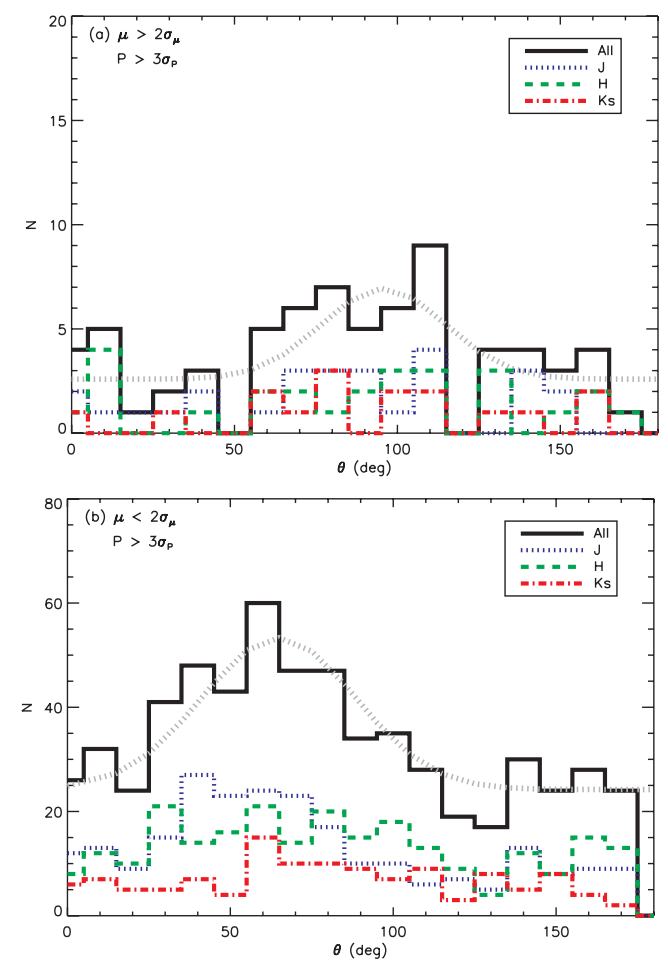

Fig. 10. - (a) Histograms of the polarization angle of the sources which have proper motion $\mu>2 \sigma_{\mu}$ and $P>3 \sigma_{P}$ for the $J, H$, and $K_{s}$ bands. (b) Histograms of the polarization angle of the sources which have proper motion $\mu<2 \sigma_{\mu}$ and $P>3 \sigma_{P}$ for the $J, H$, and $K_{s}$ bands.

Intermediate-Mass, Pre-Main-Sequence Population in The 30 Doradus Nebula, AJ, 122, 858

Davis, L. J., \& Greenstein, J. L. 1951, The Polarization of Starlight by Aligned Dust Grains, ApJ, 114, 206 
Dolginov, A. Z., \& Mytrophanov, I. G. 1976, Orientation of Cosmic Dust Grains, Ap\&SS, 43, 291

Gaensler, B. M., Haverkorn, M., Staveley-Smith, L., Dickey, J. M., McClure-Griffiths, N. M., Dickel, J. R., \& Wolleben, M. 2005, The Magnetic Field of the Large Magellanic Cloud Revealed through Faraday Rotation, Science, 307, 1610

Haynes, R. F., et al. 1991, A Radio Continuum Study of the Magellanic Clouds, A\&A, 252, 475

Isserstedt, F., \& Reinhardt, M. 1976, On The Structure of The Magnetic Field in The Large Magellanic Cloud, MNRAS, 176, 693

Kandori, R., et al. 2006, SIRPOL: a JHK ${ }_{s^{-}}$ Simultaneous Imaging Polarimeter for the IRSF 1.4m Telescope, Proc. SPIE, 6269, 159

Kandori, R., et al. 2007, Near-Infrared Imaging Polarimetry of the Star-Forming Region NGC 2024, PASJ, 59, 487

Kato, D., et al. 2007, The IRSF Manellanic Clouds Point Source Catalog, PASJ, 59, 615

Kepley, A. A., Mühle S., Wilcots, E. M., Everett, J., Zweibel, E., Robishaw, T., \& Heiles, C. 2007, Magnetic Fields in Irregular Galaxies, IAUS, 259, 555

Kim, S., et al. 2010, Colod Dust Clumps in Dynamically Hot Gas, A\&A, 518, L75

Kwon, J., Choi, M., Pak, S., Kandori, R., Tamura, M., Nagata, T., \& Sato, S. 2010, Magnetic Structure of The HH 1-2 Region: Near-Infrared Polarimetry of Point-Like Sources, ApJ, 708, 758

Lazarian, A. 2006, Grain Alignment, Polarization and Magnetic Fields, A\&AS, 8049

Maercker, M., \& Burton, M. G. 2005, L-band (3.5 $\mu \mathrm{m})$ IR-excess in massive star formation I. 30 Doradus, A\&A, 438, 663

Mathewson, D. S., \& Ford, V. L. 1970, The MagneticField Structure of the Magnellanic Clouds, ApJ, 160, L43

Nakajima, Y., et al. 2007, First NIR Polarimetry of 30 Doradus, PASJ, 59, 519

Nagayama, T., et al. 2003, SIRIUS: a Near Infrared Simultaneous Three-Band Camera, Proc. SPIE, 4841, 459

Nikolaev, S., \& Weinberg, M. D. 2000, Stellar Populations in the Large Magellanic Cloud from 2MASS, ApJ, 542, 804

Ostriker, E. C., Stone, J. M. \& Gammie, C. F. 2001, Density, Velocity, and Magnetic Field Structure in Turbulent Molecular Cloud Models, ApJ, 546, 980

Pak, S., Jaffe, D. T., van Dishoeck, Ewine F., Johansson, L. E. B., \& Booth, R. S. 1998, Molecular Cloud Structure in the Magellanic Clouds: Effect of Metallicity, ApJ, 498, 735
Parthasarathy, M., \& Jain, S. K. 1993, UNVRI Polarization Measurements of POST AGB Stars, IAUS, 155,353

Schmidt, Th. 1970, Polarization Measurements and Magnetic Field Structure within the Magellanic Clouds, A\&A, 6, 294

Schmidt, Th. 1976, Starlight Polarization in the Magellanic Cloud Regions, A\&A, 24, 357

Scowen, P., et al. 2009, The Magellanic Clouds Survey: a Bridge to Nearby Galaxies, Astro, 266

Stetson, P. B. 1987, DAOPHOT: A Computer Program for Crowded-Field Stellar Photometry, PASP, 99, 191

Tinbergen, J. 1996, Dichroism, in Astronomical Polarimetry (Boston: Cambridge Univ. press), 42

Vieira, K., et al. 2010, Proper Motion Study of the Magellanic Clouds using SPM material, AJ, 140, 1934

Wardle, J. F. C., \& Kronberg, P. P. 1974, The Linear Polarization of Quasi-Stellar Radio Sources at 3.71 and 11.1 centimeters, ApJ, 194, 249

Walborn, N. R., \& Blades J. C. 1997, Spectral Classification of The 30 Doradus Stellar Populations, ApJS, 112,457

Wayte, S. R. 1990, Structure of the Interstellar Medium in the Magellanic Clouds, ApJ, 355, 473

Wielebinski, R. 1995, Galactic and Extragalactic Magnetic Fields, Reviews in Modern Astronomy, Vol. 8, Reviews in Modern Astronomy, ed. G. Klare, 185200 